This is the accepted version of the article:

Roche, S.; Valenzuela, S.O.. Graphene spintronics: Puzzling controversies and challenges for spin manipulation. Journal of Physics D - Applied Physics, (2014). . : - .

10.1088/0022-3727/47/9/094011.

Available at:

https://dx.doi.org/10.1088/0022-3727/47/9/094011 


\title{
Graphene Spintronics: Puzzling Controversies and Challenges for Spin Manipulation
}

\author{
Stephan Roche and Sergio O. Valenzuela \\ ICREA - Institució Catalana de Recerca i Estudis Avançats, 08010 Barcelona, Spain \\ ICN2 - Institut Catala de Nanociencia i Nanotecnologia, Campus UAB, Bellaterra, \\ 08193 Barcelona, Spain \\ E-mail: stephan.roche@icn.cat, SOV@icrea.cat
}

\begin{abstract}
This article presents the current puzzling controversy between theory and experimental results concerning the magnitude and mechanisms leading to spin relaxation in graphene-based materials. On the experimental side, it is surprising that regardless of the quality of the graphene monolayer, which is characterized by the carrier mobility, the typical Hanle precession measurements yield spin diffusion times $\left(\tau_{s}\right)$ in the order of $\tau_{s} \sim 0.1-1 \mathrm{~ns}$ (at low temperatures), which is several orders of magnitude below the theoretical estimates based on the expected low intrinsic spinorbit coupling in graphene. The results are weakly dependent on whether graphene is deposited onto $\mathrm{SiO}_{2}$ or boron-nitride substrates or suspended, with the mobility spanning 3 orders of magnitude. On the other hand, extraction from two-terminal magnetoresistance measurements, accounting for contact effects result in $\tau_{s} \sim 0.1 \mu \mathrm{s}$, and corresponding diffusion lengths of about $100 \mu \mathrm{m}$ up to room temperature. Such discrepancy jeopardizes further progress towards spin manipulation on a lateral graphene two-dimensional platform. After a presentation of basic concepts, we here discuss state-of-the-art literature and the limits of all known approaches to describe spin transport in massless-Dirac Fermions, in which the effects of strong local spinorbit coupling ceases to be accessible with perturbative approaches. We focus on the limits of conventional views of spin transport in graphene and offer novel perspectives for further progress.
\end{abstract}


Graphene Spintronics: Puzzling Controversies and Challenges for Spin Manipulation 2

As highlighted in the International Technology Roadmap for Semiconductors (ITRS), devices relying on spintronics (that use the spin degree of freedom and magnetism inherent non-volatility) hold unique prospects for Information and Communication Technologies. Amongst potential channels for spintronics, graphene, already acclaimed for its potential for more-than-Moore electronics, is very promising. Indeed, graphene could offer true capability for efficient spin manipulation and for the creation of a full spectrum of spintronic nanodevices for beyond CMOS while being compatible with more-than-Moore CMOS and non-volatile low energy MRAM memories [1]. Ultra low energy rewritable microchips, transistors and logic gates, including information storage and processing on a common circuit platform could be envisioned. However, while long spin transport in graphene has been demonstrated $[2,3]$, the reported spin diffusion times remain several orders of magnitude lower than theoretically predicted [4], whereas the related sources for spin dephasing and scattering remain debated in the literature.

Spin-orbit coupling in graphene is expected to be weak first because of the low atomic number carbon $\left(Z=6\right.$, while spin-orbit interaction scales as $\left.Z^{4}\right)$. Moreover, the natural occurrence of zero nuclear spin isotope ${ }^{12} \mathrm{C}$ is close to $99 \%$ and makes hyperfine interaction a vanishingly small decoherence mechanism. Theoretical calculations show that clean graphene exhibits a very low intrinsic (intra-atomic) spin-orbit coupling $\lambda_{I} \sim 12 \mu \mathrm{eV}$, with a related spin-split gap of about $25 \mu \mathrm{eV}$ (which can be derived using a tight-binding Slater-Koster model $[5,6]$ ), whereas the application of an external electrical field (perpendicular to the graphene layer) results in gap-closing. Such low spin-orbit coupling should produce relaxation times in the microsecond scale.

Experiments at room temperature on spin injection in monolayer graphene on $\mathrm{SiO}_{2}$ substrates $[2,7,8,9,10,11,12]$ report relatively short spin-relaxation times (in the order of $1 \mathrm{~ns}$ ), several orders of magnitude lower than the original theoretical predictions. Proposals to explain the unexpectedly short spin relaxation lengths include spin decoherence due to interactions with the underlying substrate, the presence of random distribution of impurities and the adsorption of molecules, the generation of ripples or corrugations, the presence of strain, topological lattice disorder, graphene edges, etc (see for instance Ref. [11]). Experimental results show that the type of dielectric [2,13], the impedance of the contacts[14], enhanced spin-flip processes,[11] or even the absence of a substrate $[15,16]$ do not seem to affect the spin-relaxation times very significantly.

The nature of spin relaxation is actually a fundamental debated issue. Following what is known for metals and semiconductors, two mechanisms have been considered in graphene, namely the Elliott-Yafet (EY) type and the Dyakonov-Perel (DP) mechanism $[17,18]$. The EY mechanism has been derived for spin relaxation in metals, and relates the spin dynamics with electron scattering off impurities or phonons. Each scattering event changes the momentum, with a finite spin-flip probability, which is derived by a perturbation theory (assuming weak spin-orbit scattering). This gives rise to weakantilocalization phenomena in the low temperature regime, and to a typical scaling 
Graphene Spintronics: Puzzling Controversies and Challenges for Spin Manipulation 3

(a)

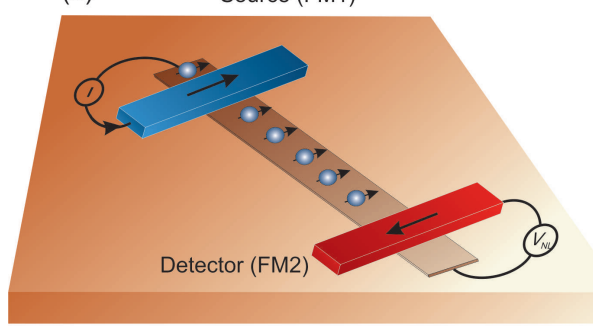

(b)

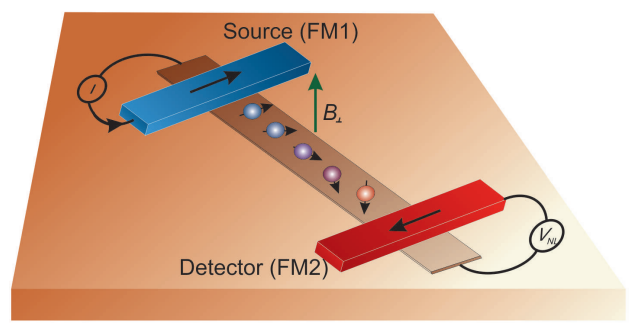

(c)

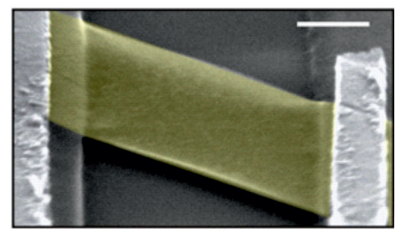

(d)

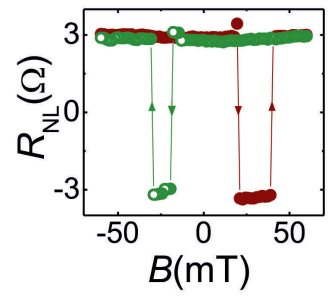

(e)

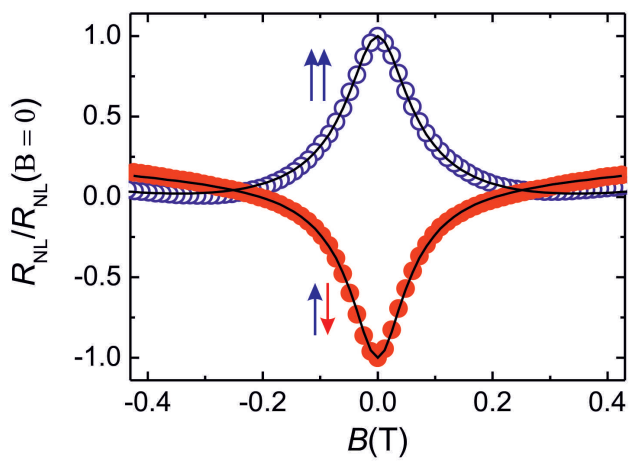

Figure 1. (a) Scheme of a nonlocal spin injection detection device. An in-plane magnetic field is applied along the ferromagnets to change the relative orientation of the ferromagnet magnetizations (b) Electrical detection of spin precession in a perpendicular magnetic field. (c) Scanning electron microscope image of a suspended graphene device. Only the two inner contacts in (a) are visualized. The white bar represents $200 \mathrm{~nm}$. (d) Nonlocal measurements in a graphene device using the configuration shown in (a) as a function of the magnetic field. (d) Spin precession measurements in the configuration shown in (b) for the device in (d) for parallel and antiparallel configuration of the magnetizations of the electrodes. Adapted from Ref. [16].

behavior of the spin relaxation time with momentum relaxation as $\tau_{s}^{E Y} \sim \alpha \tau_{p}$. The DP mechanism is an efficient mechanism of spin relaxation due to spin orbit coupling in systems lacking inversion symmetry. Examples of materials without inversion symmetry include semiconductors from groups III-V (e.g. GaAs) or II-VI (e.g. ZnSe), where inversion symmetry is broken by the presence of two distinct atoms in the Bravais lattice. Electron spins precess along a magnetic field which depends on the momentum. At each scattering event, the direction and frequency of the precession changes randomly. The scaling behavior is opposite to the EY mechanism, $\tau_{s}^{D P} \sim \hbar^{2} /\left(\lambda_{R}^{2} \tau_{p}\right)$. The most recent theoretical derivation in monolayer graphene (taking into account the Dirac cone physics) reports on some variation of the scaling as $\tau_{s} \sim \epsilon_{F}^{2} \tau_{p} / \lambda_{R}^{2}$ which is of the EYtype[19]. Such result is derived assuming an absence of intervalley scattering and by treating the spin-orbit coupling perturbatively. However, the corresponding estimation of spin relaxation times still remains several orders of magnitude too long compared to 
experiments, demanding for more generalized and non-perturbative treatments of spin dephasing phenomena in complex and disordered graphene materials.

(a)

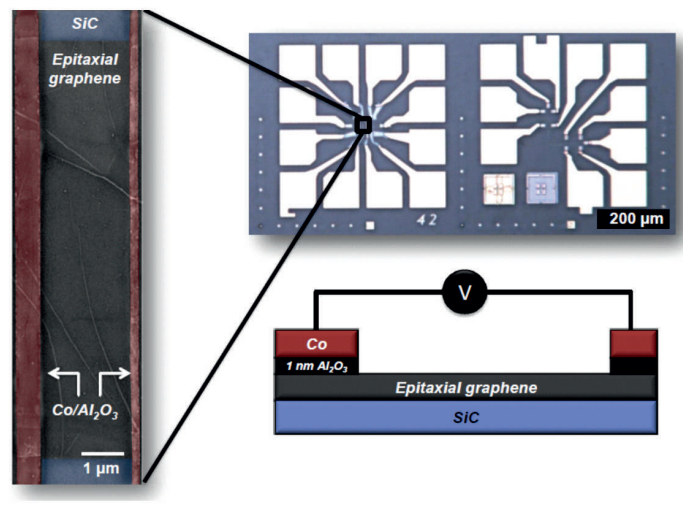

(b)

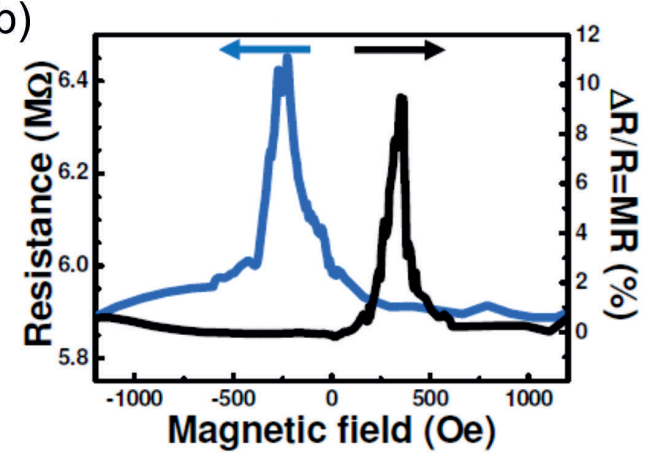

Figure 2. (a) Scanning electron microscope image of a two-terminal local spin valve (left). The width of the epitaxial graphene channel on $\mathrm{SiC}$ is $10 \mu \mathrm{m}$, and the distance between the two $\mathrm{Al}_{2} \mathrm{O}_{3} / \mathrm{CO}$ electrodes is $L=2 \mu \mathrm{m}$. Optical image of the entire structure, including contact pads (right). (b) Large local $\Delta R$ spin signals measured at 4 K. Adapted from Ref. [3].

In Ref.[12], CVD-grown monolayer and bilayer graphene samples were compared, with the surprising result of EY-type and DP-type scalings in monolayer and bilayer graphene, respectively. Typical transport time scales were found to be $\ell_{e}=v_{F} \times \tau_{p} \sim$ $20-30 \mathrm{~nm}$, with $\tau_{s} \sim 175-230$ ps for the monolayer case, and $\ell_{e}=v_{F} \times \tau_{p} \sim 30-50$ $\mathrm{nm}, \tau_{s} \sim 260-340 \mathrm{ps}$ for the bilayer case. Both types of samples exhibited carrier mean free paths of a few tens of nanometers, and spin relaxation times of similar magnitude, but with different scaling behaviors.

The estimation of the spin relaxation time (as well as the spin diffusion coefficient) is generally achieved through spin valve measurements and Hanle precession effects (Figs. $1 \mathrm{~b}$ and 1e), which are non-local transport measurements in which the spin diffusion far from the source/drain contact is tuned with an external and perpendicular magnetic field, inducing spin precession [20, 21, 22]. The basic physical principles of the nonlocal device are the electrical spin injection, the generation of nonequilibrium spin accumulation, and the electrical spin detection using ferromagnetic electrodes as spin polarizers. A sketch of the device is shown in Fig. 1a. Fig. 1c shows an scanning electron image of a detail of an actual device based on a suspended graphene flake [16]. An injected current $I$ on the source (FM1) generates spin accumulation in graphene, which is quantified by the detector voltage $V_{N L}$. The current $I$ is injected from FM1 and away from FM2. Electron spins diffuse isotropically from the injection point, and the sign of $V_{N L}$ is determined by the relative magnetization orientations of FM1 and FM2 (Fig. 1d). The spin accumulation, and $V_{N L}$, can be quantified from the spin splitting in the electrochemical potential induced by spin injection, which decays over a characteristic length $\lambda_{s}$. 
The spin direction can be manipulated by inducing a coherent spin precession induced by an applied magnetic $B_{\perp}[20,21,22]$ which is perpendicular to the substrate (Fig. 1b). In this situation, the spins that are polarized along the FM1 magnetization rotate around an axis that is parallel to the field with a period determined by the Larmor's frequency $\Omega=\gamma B_{\perp}$, where $\gamma$ is the gyromagnetic ratio of the electron. During the time $t$ that it takes the electron to travel to FM2, the spin will rotate a certain angle $\phi$ given by $\phi=\gamma t$. Because $V_{N L}$ is sensitive to the projection of the spins along the FM2 magnetization, it oscillates as a function of $B_{\perp}$ (Figs. 1b and 1e). The measured non-local magnetoresistance $R_{N L}=V_{N L} / I$ is usually modelled with a onedimensional spin-Bloch diffusion equation[20, 21, 22] which assumes a diffusive (random walk) propagation of spin, and relate the resistance to microscopic parameters through

$$
R_{N L} \sim \int_{0}^{+\infty} \frac{1}{\sqrt{4 \pi D t}} e^{-\frac{L^{2}}{4 D_{s} t}} \cos (\Omega t) e^{-\frac{t}{\tau_{s}}}
$$

with $D_{s}=v_{F} \tau_{s}^{2}\left(\tau_{s}\right.$ the spin relaxation time) the spin diffusion coefficient, and $L$ the distance in between electrodes. An important observation is that such approach cannot tackle a situation of ballistic (or quasiballistic) spin motion, and needs further generalization particularly for describing clean graphene, for which mean free paths can be several hundreds of nanometers long [23], thus comparable to the typical electrode spacing. Additionally, for more disordered graphene, the contribution of quantum interferences and localization phenomena (which in certain materials have shown to be robust up to $100 \mathrm{~K}$ ) are neglected and could affect any estimation. Finally $\tau_{s}$ has been also estimated independently from two-terminal spin valves measurements (using a phenomenological approach), but the results turn out to be orders of magnitudes different from the obtained with Hanle measurements (Fig. 2, adapted from Refs. [3, 24]).

Even more puzzling, recent experiments on monolayer graphene on top of boronnitride substrates show that neither EY nor DP mechanisms alone allow for a fully consistent description of the spin relaxation [13]. A tentative crossover is established depending on charge density, and different processes for spin relaxation are assumed to coexist, but without addressing their respective microscopic origin. One observes that the derivation of all of these possible spin relaxation mechanisms are generally treated theoretically assuming pure bulk transport, while the contribution of tunneling resistances at interfaces between injection electrodes and graphene can vary substantially from device to device, depending on the quality and nature of material interface and conductance mismatch features.

All of these results underscore the lack of theoretical understanding of spin propagation and spin relaxation mechanisms in graphene, demanding for further theoretical inspection and more quantitative and quantum simulation of spin transport and intrinsic spin relaxation mechanisms on one side, and contact effects on the other side. In that regard, it seems necessary to develop computational approaches able to explore the regimes out of reach of perturbative treatments and phenomenology used so far. The full accounting of both spin injection properties and intrinsic spin relaxation 
phenomena in a quantum simulation would be key to disentangle both contributions and eventually obtain a comprehensive understanding of spin relaxation times in graphene devices.

Beyond such fundamental understanding of spin relaxation, another key issue that needs to be addressed is the engineering of spin gating for progressing towards the manipulation of spin currents in graphene devices. The fundamental challenge in designing spin-logic devices lies in developing external ways to control (gate) the propagation of spin-(polarized) currents at room temperature [31]. Tuning spin transport signals could be achieved by magnetic proximity effects, including the deposition of magnetic insulators (such as Europium-oxide EuO [28, 29, 30]), or the creation of local magnetic ordering [26, 27]. In a recent work, Yang and coworkers used first-principles calculations (within the GGA +U approximation) to compute the electronic interaction between graphene and a magnetic insulator (EuO) [30]. An induced spin polarization of up to $25 \%$ of graphene $\pi$-orbitals by proximity effect, together with an induced large exchange-splitting band gap of about $36 \mathrm{meV}$ were reported. Figure 3 shows the band structure of graphene on $\mathrm{EuO}$ close the Dirac cone, which is spin-split. By using external gating effect, one could thus envision to turn ON and OFF spin polarized current by shifting the Fermi level from the valence band to the conduction bands. The deposition of EuO films on graphene has been experimentally demonstrated using reactive molecular beam epitaxy in a special adsorption-controlled and oxygen-limited regime, without invasive effect on the electronic characteristics of graphene [29]. Such experimental advances and predicted spin filtering and gap opening bring the possibility for spin gating by magnetic proximity effect at a relatively high temperatures, although other magnetic insulators such as Yttrium Iron Garnet (with a Curie temperature of $550 \mathrm{~K}$ ) would be more suitable for eventually developing roomtemperature graphene spin devices.

The deposition of certain types of heavy atoms or hydrogen in graphene has been predicted to considerably enhance local spin-orbit coupling or even trigger the formation of topological insulating phases in the material $[32,33,34,35]$. The introduction of hydrogen covalently bonded to graphene results in out-of-plane distortions of the planar carbon bonds that may allow a strong enhancement in the spin-orbit interaction, which could increase from $\mu \mathrm{eV}$ up to several meV. Such increase was recently observed after the addition of a small amount of hydrogen from the dissociation of hydrogen silsesquioxane [36] . Additionally, several possibilities for generating photo-induced band-gaps and the formation of states akin to those of topological insulators have been recently reported [37, 38, 39]. Although these results have established a possible foundation for groundbreaking spin manipulation, much work remains to be accomplished to make the long-standing expected spin-based devices emerge as a reality. 


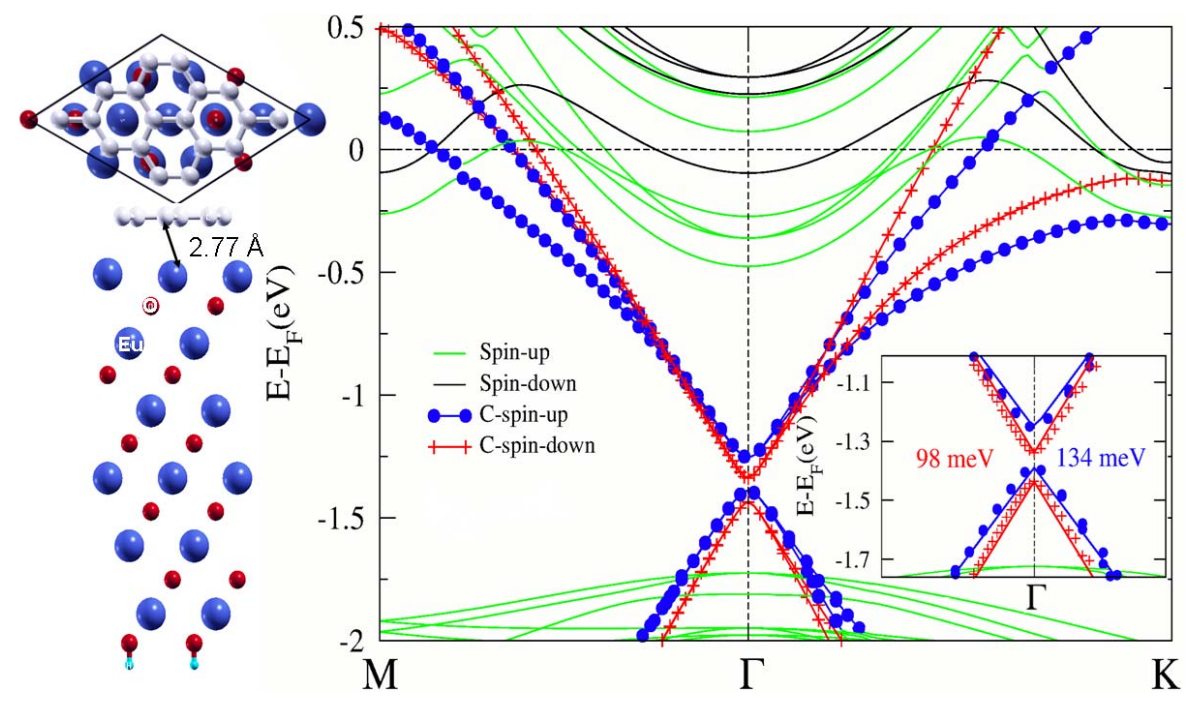

Figure 3. Side and top views of the calculated crystalline structures for graphene on top of a six bilayer $\mathrm{EuO}$ film. The bottom of $\mathrm{EuO}$ is terminated with hydrogen atoms. Band structure of graphene on $\mathrm{EuO}$. Green (blue) and black (red) represent spin up and spin down bands of EuO (graphene), respectively. Inset: zoom around the Dirac cone. Adapted from Ref. [30].

\section{Acknowledgments}

S.R an S.O.V acknowledge the Spanish Ministry of Economy and Competitiveness MINECO for funding (MAT2012-33911, MAT2010-18065).

\section{References}

[1] H. Dery, H. Wu, B. Ciftcioglu, M. Huang, Y. Song, R. Kawakami, J. Shi, I. Krivorotov, I. Zutic, L.J. Sham, Nanospintronics Based on Magnetologic Gates IEEE Trans. Electron Devices 59, 259 (2012)

[2] N. Tombros, C. Jozsa, M. Popinciuc, H.T. Jonkman, B.J. van Wees, Electronic spin transport and spin precession in single graphene layers at room temperature, Nature 448, 571-574 (2007)

[3] B. Dlubak, M.-B Martin, C. Deranlot, B. Servet, S. Xavier, R. Mattana, M. Sprinkle, C. Berger, WA De Heer, F Petroff, A Anane, P Seneor, A Fert, Highly efficient spin transport in epitaxial graphene on SiC, Nature Physics 8, 557 (2012)

[4] D. Huertas-Hernando, F. Guinea, and A. Brataas, Spin-orbit coupling in curved graphene, fullerenes, nanotubes, and nanotube caps, Phys. Rev. B 74, 155426 (2006)

[5] M. Gmitra, S. Konschuh, C. Ertler, C. Ambrosch-Draxl, and J. Fabian, Phys. Rev. B 80, 235431 (2009)

[6] S. Konschuh, M. Gmitra, and J. Fabian, Phys. Rev. B 82, 245412 (2010)

[7] N. Tombros, S. Tanabe, A. Veligura, C. Jozsa, M. Popinciuc, H. T. Jonkman, B. J. Van Wees, Anisotropic spin relaxation in graphene, Phys. Rev. Lett. 101 (4), 46601 (2008)

[8] Han, W.; Pi, K.; Bao, W.; McCreary, K. M.; Li, Y.; Wang, W. H.; Lau, C. N.; Kawakami, R. K. Appl. Phys. Lett. 94, 222109 (2009).

[9] Han, W.; Wang, W. H.; Pi, K.; McCreary, K. M.; Bao, W.; Li, Y.; Miao, F.; Lau, C. N.; Kawakami, R. K. Phys. Rev. Lett. 102, 137205 (2009). 
Graphene Spintronics: Puzzling Controversies and Challenges for Spin Manipulation 8

[10] Yang, T.-Y.; Balakrishnan, J.; Volmer, F.; Avsar, A.; Jaiswal, M.; Samm, J.; Ali, S. R.; Pachoud, A.; Zeng, M.; Popinciuc, M.; Güntherodt, G.; Beschoten, B.; Özyilmaz, B. Phys. Rev. Lett. 107, 047206 (2011).

[11] W. Han and R. K. Kawakami, Phys. Rev. Lett. 107, 047207 (2011)

[12] Ahmet Avsar T. Yang, S. Bae, J. Balakrishnan, F. Volmer, M. Jaiswal, Z. Yi, S. Rizwan Ali, G.Gütherodt, B.-H.Hong, B. Beschoten, B. Özyilmaz, Towards wafer scale fabrication of graphene based spin valve devices, Nano letters 11, 2363-2368 (2011)

[13] P. J. Zomer, M. H. D. Guimaraes, N. Tombros, and B. J. van Wees, Long-distance spin transport in high-mobility graphene on hexagonal boron nitride. Phys. Rev. B 86, R161416(R) (2012)

[14] Han, W.; Pi, K.; McCreary, K. M.; Li, Y.; Wong, J. J. I.; Swartz, A. G.; Kawakami, R. K. Phys. Rev. Lett. 105, 167202 (2010).

[15] M. H. Diniz Guimaraes, A.Veligura, P. J. Zomer, T. Maassen, I. J. Vera-Marun, N. Tombros, B. J. van Wees, Nano Lett. (2012).

[16] I. Neumann, J. Van de Vondel , G. Bridoux , M. V. Costache, F. Alzina , C. M. Sotomayor Torres , and S. O. Valenzuela, Electrical Detection of Spin Precession in Freely Suspended Graphene Spin Valves on Cross-Linked Poly(methyl methacrylate), Small 9, 156-160 (2013)

[17] I. Žutić, J. Fabian, S. Das Sarma, Spintronics: fundamentals and applications. Rev. Mod. Phys. 76,323 (2004).

[18] J. Fabian, A. Matos-Abiague, C. Ertler, P.Stano, I. Zutic, Semiconductor spintronics, Acta Physica Slovaca 57, 565 (2007)

[19] H. Ochoa, A. H. Castro Neto, F. Guinea, Phys. Rev. Lett. 108, 206808 (2012)

[20] M. Johnson and R.H. Silsbee, Phys. Rev. Lett. 55, 1790 (1985); Phys. Rev. B 35, 4959 (1987); Phys. Rev. B 37, 5236 (1988).

[21] F. Jedema, et al. Nature 416, 713 (2006)

[22] S. O. Valenzuela, Int. J. Mod. Phys. B 23, 2413 (2009).

[23] Xu Du, Ivan Skachko, Anthony Barker, and Eva Y. Andrei, Approaching ballistic transport in suspended graphene, Nature Nanotechnology 3, 491 - 495 (2008)

[24] P. Seneor, B. Dlubak, M.-B. Martin, A. Anane, H. Jaffres and A. Fert, Spintronics in Graphene, MRS Bulletin 37, 1245 - 1254 (2012)

[25] T. Maassen, J. Berg, F. Fromm, T. Seyller, R. Yakimova, B.J.v. Wees, Long Spin Relaxation Times in Wafer Scale Epitaxial Graphene on SiC(0001), Nano Letters, 12, 1498-1502 (2012)

[26] D.Soriano, N. Leconte, P. Ordejon, J.-Ch. Charlier, J. Palacios, S. Roche, Magnetoresistance and Magnetic Ordering Fingerprints in Hydrogenated Graphene, Phys. Rev. Lett. 107, 016602 (2011)

[27] K. McCreary, A. G. Swartz, W. Han, J. Fabian, K. Kawakami, Phys. Rev. Lett. 109, 186604 (2012)

[28] H. Haugen, D. Huertas-Hernando, and A. Brataas, Spin transport in proximity-induced ferromagnetic graphene, Phy. Rev. B 77, 115406 (2008)

[29] A. G. Swartz, P. M. Odenthal, Y. Hao, R. S. Ruo, and R. K. Kawakami, Integration of the Ferromagnetic Insulator EuO onto Graphene, ACS Nano. 27, 10063 (2012)

[30] H. X. Yang, A. Hallal, D. Terrade, X. Waintal, S. Roche, and M. Chshiev, Magnetic InsulatorsInduced Proximity Effects in Graphene: Spin Filtering and Exchange Splitting Gaps, Phys. Rev. Lett. 110, 046603 (2012)

[31] Y. G. Semenov, K. W. Kim, and J. M. Zavada, Spin field effect transistor with a graphene channel, Appl. Phys. Lett. 91, 153105 (2007)

[32] C. L. Kane and E.J. Mele, Z2 Topological Order and the Quantum Spin Hall Effect, Phys. Rev. Lett. 95, 146802 (2005)

[33] A. H. Castro Neto and F. Guinea, Phys. Rev. Lett. 103, 026804 (2009)

[34] H. Jiang, Z. Qiao, H. Liu, J. Shi, Q. Niu, Stabilizing Topological Phases in Graphene via Random Adsorption, Phys. Rev. Lett. 109, 116803 (2012)

[35] C. Weeks, J. Hu, J. Alicea, M. Franz, R. Wu, Engineering a robust quantum spin Hall state in graphene via adatom deposition, Phys. Rev. X 1, 021001 (2011)

[36] Balakrishnan, J,; Kok Wai Koon, G.; Jaiswal, M.; Castro Neto, A. H.; Özyilmaz, B. Nature Phys. 
Graphene Spintronics: Puzzling Controversies and Challenges for Spin Manipulation 9

9, 284-287 (2013).

[37] H.L. Calvo, H. M. Pastawski, S. Roche, and L. E. F. Foa Torres, Tuning laser-induced band gaps in graphene, Appl. Phys. Lett. 98, 232103 (2011)

[38] Z. Gu, H. A. Fertig, D. P. Arovas, and A. Auerbach, Floquet Spectrum and Transport through an Irradiated Graphene Ribbon, Phys. Rev. Lett. 107, 216601 (2011)

[39] T. Kitagawa T. Oka, A. Brataas, L. Fu, E. Demler, Transport properties of nonequilibrium systems under the application of light: Photoinduced quantum Hall insulators without Landau levels, Phys. Rev. B 84, 235108 (2011) 